\title{
Feature-based Terrain Editing From Complex Sketches
}

\author{
Flora Ponjou Tasse $^{\mathrm{a}, *}$, Arnaud Emilien ${ }^{\mathrm{b}, \mathrm{c}}$, Marie-Paule Cani ${ }^{\mathrm{b}}$, Stefanie Hahmann ${ }^{\mathrm{b}}$, Neil Dodgson $^{\mathrm{a}}$ \\ ${ }^{a}$ University of Cambridge \\ ${ }^{b}$ Laboratoire Jean Kuntzmann (Univ. Grenoble-Alpes, CNRS) and Inria \\ ${ }^{c}$ LIGUM, Dept. I.R.O., Montreal University
}

\begin{abstract}
We present a new method for first person sketch-based editing of terrain models. As in usual artistic pictures, the input sketch depicts complex silhouettes with cusps and T-junctions, which typically correspond to non-planar curves in 3D. After analysing depth constraints in the sketch based on perceptual cues, our method best matches the sketched silhouettes with silhouettes or ridges of the input terrain. A deformation algorithm is then applied to the terrain, enabling it to exactly match the sketch from the given perspective view, while insuring that none of the user-defined silhouettes is hidden by another part of the terrain. We extend this sketch-based terrain editing framework to handle a collection of multi-view sketches. As our results show, this method enables users to easily personalize an existing terrain, while preserving its plausibility and style.
\end{abstract}

Keywords: First person editing, terrain, sketch-based modelling, silhouettes

\section{Introduction}

2 Terrain is a key element in any outdoor environment. Appli3 cations of virtual terrain modelling are very common in movies, 4 video games, advertisement and simulation frameworks such as ${ }_{5}$ flight simulators. Two of the most popular terrain modelling ${ }_{6}$ methods are procedural $[1,2,3,4]$ and physics-based tech7 niques $[4,5,6,7,8,9]$. The former are easy to implement and 8 fast to compute, while the latter produce terrains with erosion 9 effects and geologically sound features. However, the lack of 10 controllability in these methods is a limitation for artists.

Sketch-based or example-based terrains have been popular 12 recently in addressing these issues $[10,11,12,13,14,15,16]$. ${ }_{13}$ However, many of these methods $[12,14,16]$ assume that the 14 user sketch is drawn from a top view, which makes shape con15 trol from a viewpoint of interest difficult. Others [10, 11, 13, 15] ${ }_{16}$ only handle a restricted category of mountains, with flat sil17 houettes. Lastly, terrains fully generated from sketches typi${ }_{18}$ cally lack details. Dos Passos et al. [17] recently presented 19 a promising approach where example-based terrain modelling 20 and a first person point-of-view sketch are combined. However ${ }_{21}$ their method does not support local terrain editing and cannot 22 handle typical terrain silhouettes with T-junctions. Moreover, ${ }_{23}$ terrain patches are often repeated which may spoil the plausi24 bility of the results from other viewpoints.

${ }_{25}$ In this work, we address the problem of intuitive shape con${ }_{26}$ trol of a terrain from a first person viewpoint, while generat27 ing detailed output that is plausible from any viewpoint. To 28 achieve the intuitive shape control goal, we stick to the sketch29 based approach, but allow the user to input complex silhouettes, 30 as those are typically used to represent terrains (see Figure 1).

*e-mail:flora.ponjou-tasse@cl.cam.ac.uk
${ }_{31}$ Our interpretation of the term "complex" is similar to the one 32 used in SmoothSketch [18], where a complex sketch is a set ${ }_{33}$ of $2 \mathrm{D}$ strokes with hidden contours and cusps. To get plausi34 ble, detailed results from any viewpoint, we focus on editing 35 an existing terrain rather than starting from scratch. This ap${ }_{36}$ proach captures the coherent small details from the existing ter${ }_{37}$ rain, while avoiding the patch blending and repetition problems 38 that are typical of example-based methods. The use of an ex${ }_{39}$ isting terrain also enables matches of sketched silhouettes with 40 plausible, non planar curves on the terrain.

${ }_{41} \quad$ In practice, the user edits the input terrain by over-sketching 42 it from a first person viewpoint. The user strokes, forming a ${ }_{43}$ graph of curves with T-junctions, represent the desired silhou${ }_{44}$ ettes for the terrain. The input terrain is then deformed such 45 that its silhouettes exactly match the strokes in the current per${ }_{46}$ spective view. This means that each stroke segment is to be ${ }_{47}$ some silhouette of the output terrain, and that no other part of 48 the deformed terrain should hide them. Previous sketch-based 49 modelling methods have successfully use feature curves to de${ }_{50}$ form surfaces $[19,20]$. Our work explores the use of terrain 51 features for sketch-based terrain editing.

${ }_{52}$ Paper contributions. This paper is an extended version of ear${ }_{53}$ lier work [21] in which we first introduced a framework for ${ }_{54}$ deforming terrain features to fit user strokes. First, sketched ${ }_{55}$ strokes are ordered by inferring their relative depth from the ${ }_{56}$ height of their end-points and from the T-junctions detected in ${ }_{57}$ the sketch. Next, features of the input terrain such as silhou${ }_{58}$ ette edges and ridges are assigned to each stroke and extended ${ }_{59}$ if necessary, to cover the length of the stroke. This assignment 60 is the solution of a minimization problem expressing the sim${ }_{61}$ ilarity between a terrain feature and a stroke in the drawing 62 plane, and the amount of deformation caused by their match${ }_{63}$ ing. The selected features then become constraints for an it- 


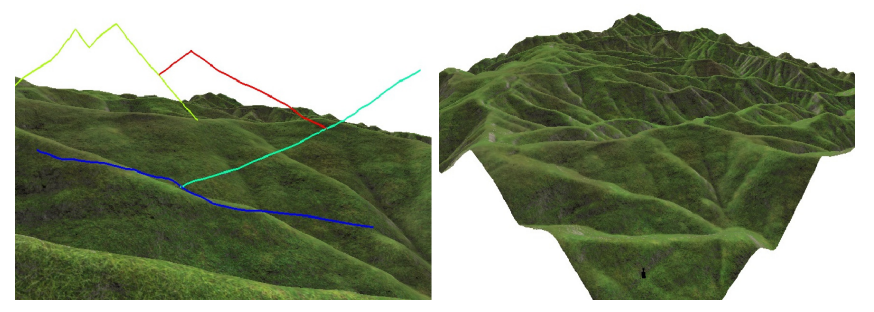

(a)

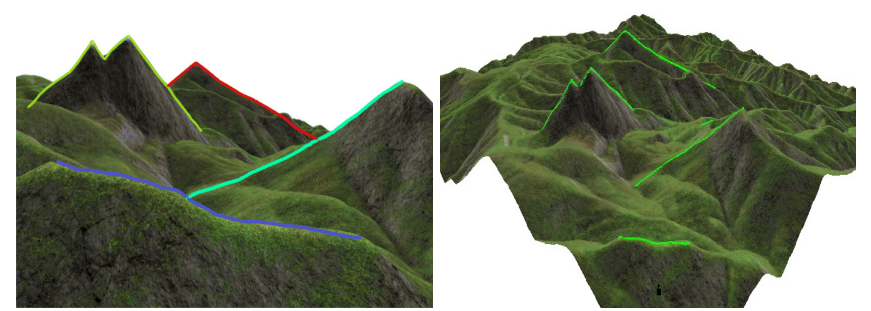

(b)

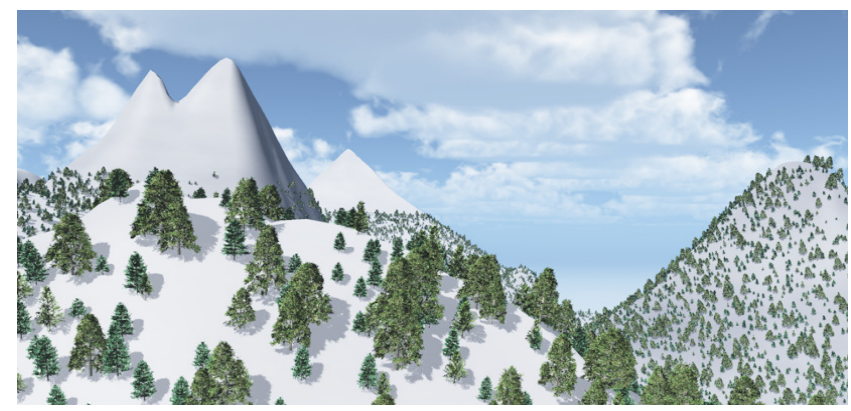

(c)

Figure 1: (a) An artist sketch (left), is used to edit an existing terrain (right). (b) Results shown from two viewpoints. Note the complex silhouettes with Tjunctions, matched to features of the input terrain. (c) shows a rendering of the resulting terrain, from a closer viewpoint.

${ }_{64}$ erative diffusion-based terrain deformation method. The main ${ }_{65}$ contributions of that earlier paper [21] are: 74 and-bound search scheme used to address the energy minimiza75 tion problem. Additionally, we propose an improved frame76 work that supports terrain editing from multi-view sketches draw 77 from different viewpoints. In the context of film making, this 78 additional tool can facilitate control of the exact shape of ter79 rain silhouettes for two or three views, which will be used for 80 key scenes. Although iteratively editing the terrain from mul${ }_{81}$ tiple viewpoints could achieve realistic landscapes, there is no 82 guarantee that silhouettes generated during one iteration will 83 not be significantly modified by subsequent iterations. The
84 stroke-to-feature matching algorithm is modified to handle all 85 sketches at once, with additional constraints that ensure that no 86 assigned feature is occluded by another. Finally, we claimed 87 in the original paper that specifically deforming terrain features 88 produces more realistic results. To illustrate this, we compare 89 the use of feature-based curve constraints in terrain deforma90 tion against using 3D planar curve constraints obtained from 91 projecting strokes on the drawing plane. We show how the two 92 types of constraints affect terrain deformation and realism on 3 93 different test cases.

95 We begin by summarising related work (Section 2). We ${ }_{96}$ then give an overview of our whole system (Section 3 ), be97 fore describing, in detail, stroke ordering (Section 4), feature 98 constraints (Section 5), terrain deformation (Section 6), and the 99 modifications needed to handle multi-view sketches from vari100 ous viewpoints (Section 7).

\section{2. Related work}

102 Most terrain modelling systems use one or a combination 103 of the following: procedural terrain generation, physics-based 104 simulation, sketch-based or example-based methods. Natali et 105 al. [22] provide a detailed survey.

106 Procedural terrain modelling methods are based on the fact 107 that terrains are self-similar, i.e. statistically invariant under 108 magnification. Fractals have the same concept of self-similarity 109 [23] and thus, fractal-based methods have been widely used in 110 terrain generation. These methods are the popular choice for 111 landscape modelling due to their easy implementation and ef112 ficient computation. They mainly consist of pseudo-randomly ${ }_{113}$ editing height values on a flat terrain by using either adaptive 114 subdivision $[1,2,3]$ or noise [2, 4]. Adaptive subdivision pro115 gressively increases the level of detail of the terrain by itera116 tively interpolating between neighbouring points and displac117 ing the new intermediate points by increasingly smaller random 118 values. Noise synthesis techniques are often preferred because 119 they offer better control. Superposing scaled-down copies of a 120 band-limited, stochastic noise function generates noise-based 121 terrains. For more information on fractal terrain generation 122 methods, see Ebert et al. [24]. Fractal-based approaches can ${ }_{123}$ generate a wide range of large terrains with unlimited level of 124 details. However, they are limited by the lack of user con125 trol or non-intuitive parameter manipulation, and the absence 126 of erosion effects such as drainage patterns. To address the ${ }_{127}$ last issue, fractal terrains can be improved using physics-based ${ }_{128}$ erosion simulation $[4,5,6,7,8,9]$. Alternatively, river net129 work generation can be incorporated in the procedural method ${ }_{130}[25,16]$. In particular, Genevaux et al. [16] create procedu$n_{131}$ ral terrains from a hydrographically and geomorphologically ${ }_{132}$ consistent river drainage network, generated from a top-view ${ }_{133}$ sketch. However, this method only captures terrains resulting 134 from hydraulic erosion, and there is no mechanism for control135 ling their silhouettes from a first person viewpoint.

Physically-based techniques generate artificial terrains by ${ }_{138}$ simulating erosion effects over some input 3D model. Mus- 
${ }_{139}$ grave et al. [4] present the first methods for thermal and hy${ }_{140}$ draulic erosion based on geomorphology rules. Roudier et al. ${ }_{141}[5]$ introduce a hydraulic erosion simulation that uses different 142 materials at various locations resulting in different interactions 143 with water. Chiba et al. [6] generate a vector field of water 144 flow that then controls how sediment moves during erosion. 145 This process produces hierarchical ridge structures and thus en146 hances realism. Nagashima [7] combines thermal and fluvial ${ }_{147}$ erosion by using a river network pre-generated with a 2D fractal 148 function. Neidhold et al. [8] present a physically correct sim149 ulation based on fluids dynamics and interactive methods that 150 enable the input of global parameters such as rainfall or local 151 water sources. Kristof et al. [9] propose fast hydraulic erosion 152 based on Smooth Particle Hydrodynamics. The main drawback ${ }_{153}$ of all these methods is that they only allow indirect user-control 154 through trial and error, requiring a good understanding of the 155 underlying physics, time and efforts to get the expected results. 156

157 Sketching interfaces and more generally feature-based edit158 ing have been increasingly popular for terrain modelling. These 159 methods can be combined with some input terrain data to gen160 erate terrains with plausible details.

Cohen et al. [10] and Watanabe et al. [11] present the first 162 terrain modelling interfaces that take as input a 2D silhouette ${ }_{163}$ stroke directly drawn on a 3D terrain model. They only han${ }_{164}$ dle a single silhouette stroke, interpreted as a flat feature curve. ${ }_{165}$ McCrae and Singh [26] use stroke-based input to create paths 166 which deform terrains. However user strokes are interpreted 167 as path layouts and not as terrain silhouettes. Multi-grid diffu168 sion methods enable generation of terrains that simultaneously 169 match several feature curves, either drawn from a top view [14] 170 or from an arbitrary viewpoint [27]. The main limitation is that 171 generated terrains typically lack realistic details.

In contrast, Zhou et al. [12] use features (actually, sketch 173 maps painted from above) to drive patch-based terrain synthe- 227 174 sis from real terrain data. Closer to our concerns, Gain et al. 228 175 [13] deform an existing terrain from a set of sketched silhou- 229 176 ettes and boundary curves. The algorithm deforms the terrain 177 based on the relative distance to the feature-curves in their re178 gion of influence, and on wavelet noise to add details to the 179 silhouettes. In this work we rather use a diffusion-based defor180 mation method to propagate feature constraints, avoiding the ${ }_{181}$ need for boundary curves. Lastly, Tasse et al. [15] present a 182 distributed texture-based terrain generation method that re-uses 183 the same sketching interface. Unfortunately, all these meth184 ods interpret each sketched silhouette as a planar feature curve, 185 which reduces the realism of the result.

186

187 Dos Passos et al. [17] propose a different approach to ad188 dress this issue. Given a set of sketched strokes drawn from 189 a first person point-of-view, copies of an example terrain are 190 combined such that the silhouettes of the resulting terrain match 191 the strokes. This gives a realistic, varying depth to silhouettes. 192 To achieve this, the algorithm assumes each stroke represents 193 a terrain silhouette. A stroke is matched with a portion of a 194 silhouette, selected from a set of silhouettes viewed from sev195 eral standing viewpoints around the example terrain. Terrain
196 slices representing portions of matched silhouette are cut from 197 the example terrain and then combined through a weighted sum 198 to produce a smooth terrain. A drawback of this method is that 199 it does not handle complex sketches with T-junctions, which 200 are common in landscape drawings. Moreover, the matching 201 process may select the same silhouette portions for different 202 strokes, thus producing unrealistic repeating patterns in the fi203 nal result. Finally, the weighted sum function used for merging 204 may fail to remove the boundary seams produced by combining 205 different terrain slices. In this work, we address these issues 206 by presenting a sketch-based method that handles T-junctions 207 in complex sketches and deforms an input terrain to match the 208 sketch rather than copy-pasting parts of it.

\section{3. Overview}

210 Let us describe our processing pipeline. As in many terrain 211 modelling and rendering methods, our terrains are represented 212 by a height field, implemented as a greyscale image storing 213 elevation values. This representation cannot emulate features ${ }_{214}$ such as overhangs and caves, but it is the most prevalent for215 mat in terrain generation because of its simplicity and efficient 216 use of storage space. For rendering purposes and silhouette de217 tection, a 3D triangular mesh is constructed from the height 218 field by connecting adjacent terrain points $(x, y, \operatorname{altitude}(x, y))$. ${ }_{219}$ Users are able to navigate on a 3D rendering of the existing 220 terrain, possibly flat, with a first-person camera always at a 221 standing viewpoint. A sketch is created by drawing one or 222 multiple strokes from the same camera position. The drawn ${ }_{223}$ strokes represent silhouettes that the artist wishes to be visible 224 from that position. Our main goal is to deform the terrain such 225 that these user constraints are respected. The following require226 ments should be satisfied:

- Every sketched stroke should be a terrain silhouette, in the current perspective view from the first-person camera viewpoint.

- Each of these terrain silhouettes should be visible, i.e. not hidden by any other part of the terrain.

- The deformed terrain should not have artifacts nor contain unrealistic deformations, from any other viewpoint.

Our solution consists of five steps, illustrated in Figure 2.

Stroke ordering: We order strokes according to their depth, ${ }_{236}$ from front to back with respect to the camera position. This ${ }_{237}$ order is used when we generate constraints for terrain deforma238 tion, so that a curve constraint is not occluded by another, when 239 viewed from the first-person viewpoint.

Feature detection: Terrain features such as silhouettes and 241 ridges are detected. Deforming existing terrain features to match 242 the desired silhouettes results in a more realistic terrain since no ${ }_{243}$ extra features are added and thus, the nature of the existing ter244 rain is best preserved.

Stroke-feature matching: For each stroke, we select a ter246 rain feature that will be deformed to fit the stroke, when seen 247 from the camera position. These deformed features represent 


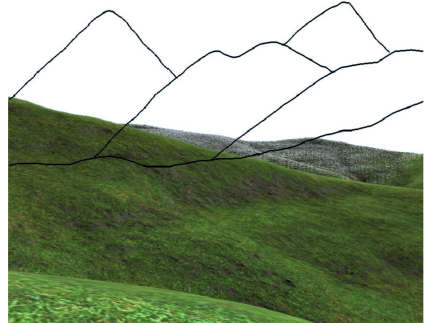

(a) User 2D sketch, in a 3D interface

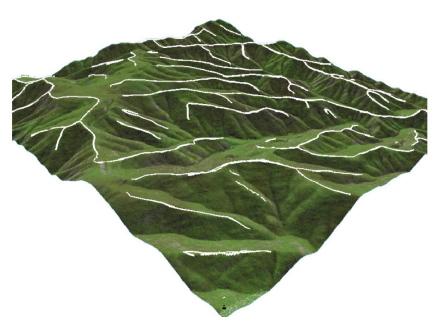

(c) Terrain feature detection (3/4 view)

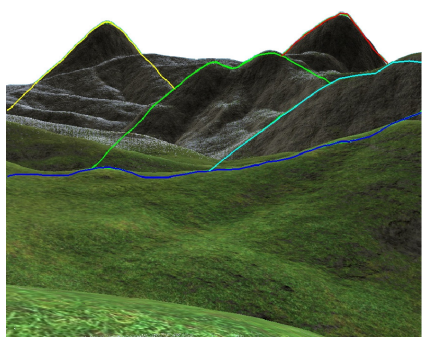

(e) Deform with matched features

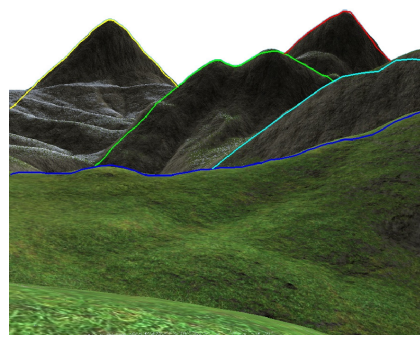

(g) Lowering protruding silhouettes

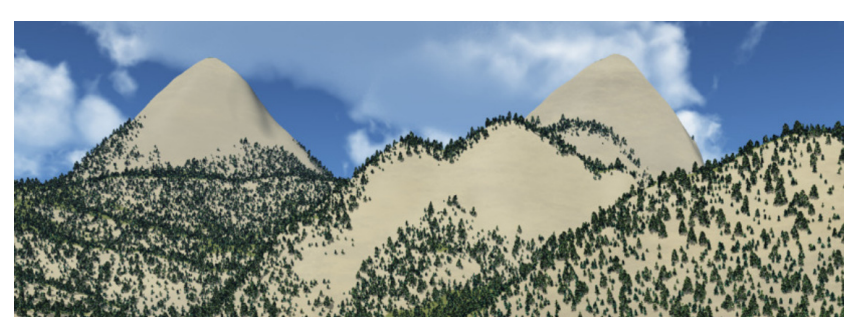

Figure 2: Overview of our terrain editing framework. (a) Unlabeled user sketch In (b), stroke colour indicates stroke ordering: blue indicates that a stroke is closer to the camera position and red indicates that it is the furthest. (c) illustrates detected features in white and (d) shows the subset of features that are assigned to user strokes. In (e,f) the terrain features are deformed so that they match the strokes from the user viewpoint. The final result in $(\mathrm{g}, \mathrm{h})$ is obtained after removing some residual artifacts.
248 the positional constraints that we use in the diffusion-based ter${ }_{249}$ rain deformation. A key idea of our framework is the expres250 sion of this feature selection step as an energy minimization 251 problem, in which we penalize features with large altitude dif252 ferences compared to their corresponding stroke as well as fea253 tures that would result in too large deformations.

Terrain deformation: We use a multi-grid Poisson solver 255 for diffusion-based terrain deformation. It solves for altitude 256 differences instead of absolute terrain positions, thus preserving 257 the small-scale features of the input terrain.

${ }_{258} \quad$ Lowering protruding silhouettes: After terrain deforma259 tion, other parts of the terrain may hide the user-specified sil260 houettes. To address this issue, we run the following iterative ${ }_{261}$ process: we detect terrain silhouettes that do not fit any user ${ }_{262}$ stroke and yet hide one of the sketched silhouettes. Extra de263 formation constraints are constructed to enforce lowering these 264 protruding silhouettes until the user-sketched silhouettes are no 265 longer occluded. The terrain is deformed with a combination of 266 previous constraints and the newly constructed constraints. We 267 repeat this process until there is no longer protruding silhouette.
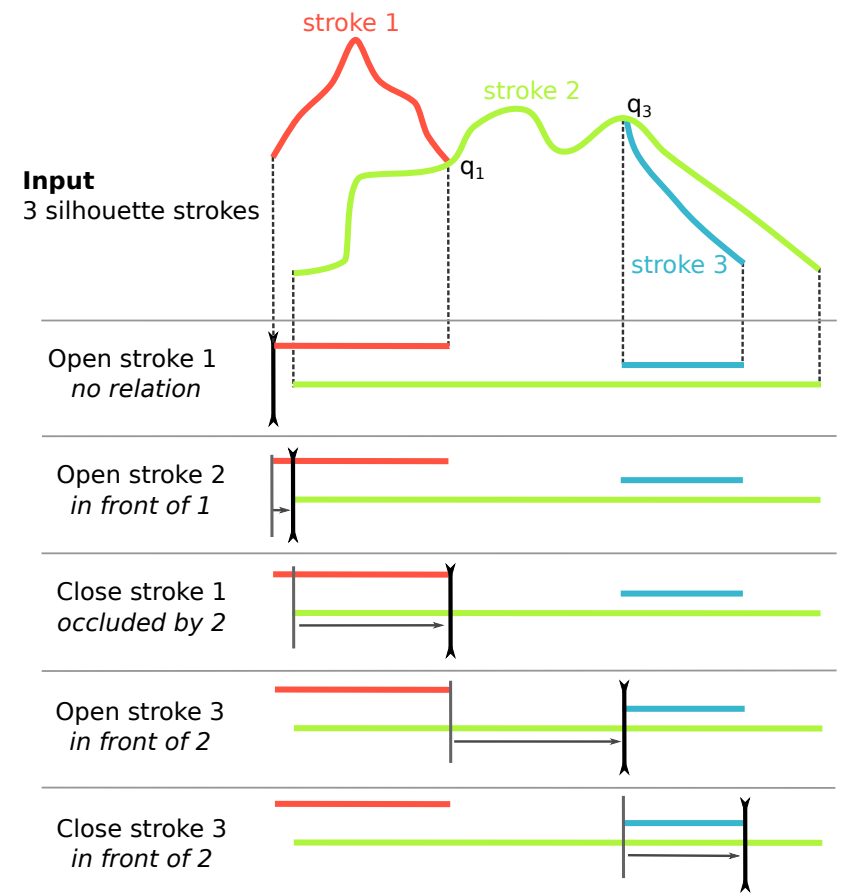

Figure 3: An input sketch (top) and the different steps of the sweeping algorithm used for scanning the sketch, labelling T-junctions and ordering strokes (bottom). As a result, stroke 3 is detected to be in front of stroke 2, which is itself in front of stroke 1. Note that the stroke colouring at the top is for illustration purposes only, the input sketch being unlabeled.

\section{4. Analysing complex terrain sketches}

269 In this section, we explain how depth ordering of silhouette 270 strokes is extracted from the user sketch.

271 The different silhouette strokes in the input sketch first need 272 to be ordered, in terms of relative depth from the camera view273 point. This is necessary since the input strokes are not labeled 274 and thus there no information of the order in which they should 
275 be processed. This will enable us to ensure, when they are 276 matched with features, that they will not be hidden by other 277 parts of the terrain. Our approach to do so is based on two ob278 servations:

- If, in the viewing plane, a silhouette lies above another, it obviously corresponds to a mountain $A$ farther away from the viewpoint than the other mountain $B$. Otherwise $A$ would hide $B$. Using height coverage for ordering them in depth is however not sufficient, since some strokes may overlap in height, as for the green and blue strokes in Figure 3.

- Furthermore, the terrain being a height field, the projection of each stroke onto the horizon ( $x$-axis of the viewing plane) is injective (no more than one height value per point).

290

These two observations allow us to solve the relative stroke 291 ordering problem using our new sweeping algorithm (see Fig292 ure 3): We consider the projections of all the strokes onto the 293 horizontal $x$ axis (depicted in the bottom part of the Figure) 294 and sweep from left to right, examining the extremities (start295 ing and endpoints in sweeping direction) and junction points of 296 the silhouette strokes. While doing so, we label the strokes' 297 extremities and the junction points in the following way: an ex298 tremity $q_{s}$ of stroke $s$ is a T-junction if its closest distance to an299 other stroke $r$ is smaller than a threshold. Information about the 300 junction point of two strokes is used to unambiguously decide 301 which stroke is occluded and thus, further from to the camera. ${ }_{302}$ An endpoint $q_{s}$ is labelled (occluded-by, $r$ ) if the oriented an${ }_{303}$ gle, measured counterclockwise, between the tangent ${ }^{1}$ of $s$ at $q_{s}$ 304 and the tangent of $r$ at $q_{s}, \angle\left(t_{s}, t_{r}\right)<180^{\circ}$. This indicates that $s$ 305 is occluded by, and thus behind, $r$. Otherwise, $s$ is in front of $r$ 306 and we label $q_{s}$ as (in-front-of, $r$ ).

${ }_{307}$ In the absence of T-junctions, stroke ordering is determined 308 using the height values at extremeties. First, we check if once 309 both strokes are projected on the horizontal axis, the interval ${ }_{310}\left[r_{\text {right }}, r_{\text {left }}\right]$ is a subset of $\left[s_{\text {right }}, s_{\text {left }}\right]$. If this is the case, we say ${ }_{311}$ that the projection of $s$ completely contains the projection of $r$ 312 and $s$ is behind $r$. Otherwise, the stroke with the lowest height ${ }_{313}$ is considered closer to the camera and thus, $s$ is behind $r$ if the 314 smallest height value of $s$ 's endpoints is larger than the smallest 315 height value of $r$ 's endpoints.

While scanning the sketch from left to right, we insert each 317 stroke in a sorting structure, at a relative depth position deter318 mined by the cues above. This results in a relative ordering of ${ }_{319}$ the user strokes.

\section{${ }_{320}$ 5. Positioning strokes in world space}

321 The key idea of our approach is to create a 3D terrain that 322 matches the user drawing, by deforming an existing one. More

\footnotetext{
${ }^{1}$ Strokes are always oriented clockwise. Hence, stroke tangents are independent of the direction in which the stroke was sketched. When labelling a starting point $q_{s}$ as T-junction, we flip its tangent.
}

${ }_{223}$ precisely, we deform the features of the existing terrain, like its 324 ridge lines, to match the user silhouette strokes. Because a ter325 rain has many features, we first have to compute to which one of 326 them it is the most appropriate to apply a deformation. In this ${ }_{327}$ section, we detail how we compute the set of terrain features 328 (Section 5.1), how we allocate one of them to each of the user 329 strokes (Section 5.2) and we present a feature completion algoззо rithm that infers the hidden parts of the silhouettes, enabling a ${ }_{331}$ more realistic terrain deformation result (Section 5.3).

\section{5.1. Feature detection: silhouettes and ridgelines}

ззз Silhouette detection on the existing terrain is based on a 334 common and naive algorithm for computing the exact silhou${ }_{335}$ ettes of a 3D mesh. Silhouette edges are detected by finding all 336 visible edges shared by a front face and a back face in the cur337 rent perspective view. Neighbouring silhouette edges are then ${ }_{338}$ linked to form long silhouette curves.

339

340 Ridge detection is based on the profile-recognition and polygon341 breaking algorithm (PPA) by Chang et al. [28]. The PPA algo342 rithm marks each terrain point that is likely to be on a ridge line, ${ }_{343}$ based on the point height profile. Segments, forming a cyclic ${ }_{344}$ graph, connect adjacent candidate points. Polygon-breaking re345 peatedly deletes the lowest segment in a cycle until the graph 346 is acyclic. Finally, the branches on the produced tree structure 347 are reduced and smoothed. The result is a graph where nodes 348 are end points or branch points connected by curvilinear ridge349 lines. An improvement of the PPA algorithm connects all the 350 terrain points into a graph using a height-based or curvature351 based weighting and computes the minimum spanning tree of 352 that graph [29]. Because we are mainly concerned with perfor353 mance and detection of large-scale ridges, we simply connect 354 candidate terrain points as in the original PPA algorithm and 355 replace the polygon-breaking with a minimum spanning forest 356 algorithm.

\section{5.2. Stroke - Feature matching}

358 In this section, we discuss a method for determining, for 359 each stroke, the terrain features which can be used to construct 360 deformation curve constraints. Viewed from the first person ${ }_{361}$ camera, these curve constraints should match the user-sketched 362 strokes. To achieve this, we first construct a feature priority 363 list for each stroke and then select features for each priority list 364 such that the sum of their associated cost is minimized.

\section{5.2.1. Feature priority list per stroke}

${ }_{366}$ For a stroke $s$, we project all terrain features on the sketch367 ing plane (i.e. we use the $2 \mathrm{D}$ projection of the feature from the 368 first-person viewpoint) and select feature curves that satisfy the 369 following condition: the $x$ interval they cover matches the one 370 of the stroke $s$. We deform the selected feature curves, and ${ }_{371}$ if necessary extend their endpoints, such that viewed from the 372 camera position, they cover the length of $s$. This deformation is 373 simply achieved by displacing the feature curve points accord374 ing to their projection on the 2D stroke in the sketching plane, 375 and their distance to the camera position. Let $f$ be a terrain fea376 ture and $f_{p}$ its projection on the stroke plane. We sweep $s$ from 
377 one extremity to another with a vertical line and sections of $f$ 378 whose projection on $f_{p}$ never intersect this line are removed. 379 Moreover, for each point $q \in f$, its altitude is modified as fol380 lows:

$$
{ }_{381} \quad q . z=q . z+k\left\|q_{p}-q_{p}^{s}\right\| \frac{\left\|q-p_{c}\right\|}{\left\|q_{p}-p_{c}\right\|}
$$

382 where $p_{c}$ is the camera position, $k=-1$ if $f_{p}$ is below $s$ and ${ }_{38 з} k=1$ otherwise, $q_{p}$ the projection of $q$ on the stroke plane, and ${ }_{384} q_{p}^{s}$ the intersection of $s$ and the vertical line passing at $q_{p}$.

385 We used this deformed version of the feature to associate the 386 following cost $E(f, s)$ to each feature $f$ with respect to stroke $s$ :

387

$$
\begin{aligned}
& E=E_{\mathrm{dis}}+E_{\mathrm{def}}+E_{\mathrm{sam}}+E_{\mathrm{ext}} \\
& E_{\mathrm{dis}}(f)=\frac{w_{1}}{\text { CurveLength }\left(f_{p}\right)} \int_{f_{p}} h_{f_{p}} d t \\
& E_{\mathrm{def}}(f)=\frac{w_{2}}{\text { CurveLength }(f)} \int_{f} h_{f} d t \\
& E_{\mathrm{sam}}(f)=\frac{w_{3} \times \operatorname{LongestEdgeLength}(f)}{\max _{g \in \operatorname{list}(s)} \operatorname{LongestEdgeLength}(g)} \\
& E_{\mathrm{ext}}(f)=\frac{w_{4} \times \operatorname{ExtendedCurveLength}(f)}{\text { CurveLength }(f)}
\end{aligned}
$$

388 where $w_{i}$ are weights, $f_{p}$ is the projection of $f$ on the stroke 389 plane, $h_{f}$ is the altitude difference between $f$ and $f$ 's projec390 tion on the terrain, and $h_{f_{p}}$ is the altitude difference between $f_{p}$ 391 and the stroke $s$. The cost $E_{\text {dis }}$ represents the dissimilarity be392 tween $f$ and $s, E_{\text {def }}$ expresses the amount of deformation along ${ }_{393} f, E_{\text {sam }}$ penalizes features with long edges and $E_{\text {ext }}$ penalizes 394 features that were extended to fully cover $s$ when viewed from 395 the camera position. All the results shown here were generated 396 with $w_{1}=w_{2}=w_{3}=w_{4}=1.0$.

$397 \quad$ All features are sorted in a priority list according to their 398 cost. Figure 4 illustrates this process for a single stroke (in this ${ }_{399}$ simple case, the feature of minimal cost is selected).

\section{5.2.2. Energy minimization}

401 The goal here is the selection of a feature curve $f$ from 402 the priority list of each stroke $s_{i}$, to construct deformation con403 straints for terrain deformation. In addition to the feature order 404 within the different priority lists, we need to take into account 405 the depth ordering for silhouette strokes computed in Section 4. 406 Therefore, this selection process can be seen as a minimiza407 tion problem. We want to find a set of stroke-feature matches 408 such that the total cost of the assignments is minimized and the 409 assigned features respect the pre-computed stroke ordering. Let ${ }_{410} S=\left\{s_{i}: i=1, \ldots, n\right\}$ be the stroke list (ordered by depth) and $f^{i}$ ${ }_{411}$ denote a feature in the priority list $L\left(s_{i}\right)=\left\{f_{k}^{i}: k=1, \ldots, m_{i}\right\}$ for ${ }_{412}$ a stroke $s_{i}$. We are looking for $\left\{f^{i}: i \in 1 \ldots n\right\}$ such that $f^{i}<f^{j}$ ${ }_{413}$ if $i<j$ and $\sum E\left(f^{i}\right)$ is minimized. Here, $f^{i}<f^{j}$ means that $f^{i}$ 414 should not be occluded by $f^{j}$, so that all deformation curve con415 straints are visible from the first person viewpoint. We process 416 the ordered stroke list from front to back, and after each stroke, ${ }_{417}$ we remove from the priority list of the next strokes, features 418 that will be occluded if selected. We chose to process strokes

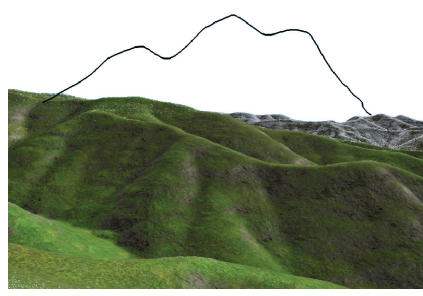

(a) User sketch

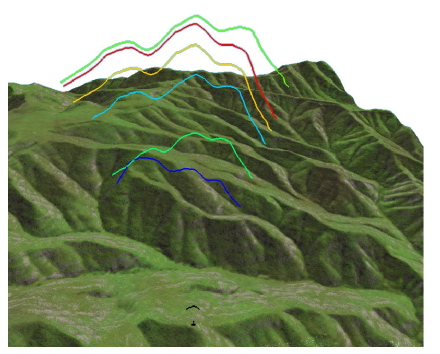

(c) Detect possible candidate matches (d) Terrain deformation using best match
Figure 4: Computing possible features to match with a user stroke. Images (a) and (d) show the terrain from the first person viewpoint used for editing, while image (b) and (c) use a higher viewpoint to better show features on the input terrain. Feature colour indicates cost: blue for the lowest cost and red for the highest.

${ }_{419}$ from front to back for two main reasons. Firstly, strokes that 420 are closest to the eye are processed first and due to $E_{\mathrm{def}}$, the ${ }_{421}$ algorithm attempts to select constraints that will minimize the 422 terrain deformation. Thus, features closer to the eye are more 423 likely to be selected. Secondly, if all the features of interest for 424 a given stroke $s_{i}$ were already selected, and therefore its priority 425 list was empty, an arbitrary curve on the terrain would be used 426 instead. If this ever occurs, we prefer it to be for background 427 silhouettes.

428 In practice, feature selections that cause any stroke to have 429 an empty priority list are penalized with a very high cost. Thus, 430 a configuration that guarantees at least one valid feature match ${ }_{431}$ for each stroke is always selected, if it exists. If no such config432 uration exists and $s_{i}$ has an empty priority list, we automatically ${ }_{433}$ compute a $3 \mathrm{D}$ embedding of the 2D stroke $s_{i}$ and use the result434 ing curve as a deformation constraint. To easily compute this ${ }_{435} 3 \mathrm{D}$ embedding, we take the two strokes lying just in front and ${ }_{436}$ just behind $s_{i}$. Then we place $s_{i}$ halfway between the terrain 437 features assigned to these two strokes. If there is no stroke re438 stricted to lie behind $s_{i}$, we place it behind the furthest stroke 439 from the viewpoint. If there is no stroke restricted to lie in front 440 of $s_{i}$, we place it in front of the closest stroke to the viewpoint. ${ }_{441}$ With this approach, each stroke is represented by a deformation 442 constraint even if it was not matched to a terrain feature during 443 the energy minimization.

444 The energy minimization problem we have described so far 445 is a NP-hard combinatorial optimization problem. Branch-and446 bound approaches are often used to overcome such computa447 tionally expensive exhaustive searches [30], since they are de- 


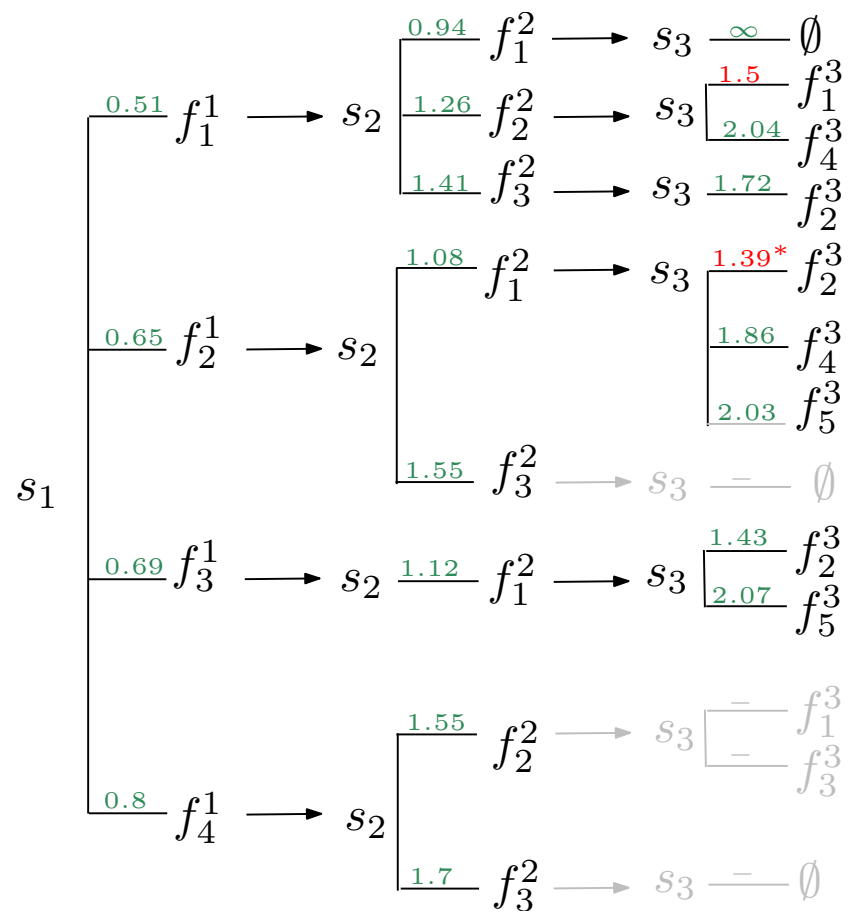

Figure 5: Energy minimization. We use a branch and bound search scheme to find the best stroke-feature matching that minimizes the total cost. Each stroke (in this example, $s_{1}, s_{2}, s_{3}$ ) has a priority list of potential candidate features, ordered from the most to the least preferable. Here $s_{1}$ has four candidates, $s_{2}$ has three and $s_{4}$ has five. Note how assigning one feature to a stroke often invalidates some features for subsequent strokes. Moreover, if a stroke no longer has a valid feature it can be assigned to, the corresponding branch has an infinite cost. Once a solution is found, branches that are guaranteed to have a cost higher than the current optimal solution are not explored (indicated in gray). The asterisk $(*)$ indicates the current best solution.

448 signed to discard non-optimal solutions early on. Here, we use 449 the branch-and-bound scheme to efficiently discard all partial 450 solutions that have a cost higher than the current best cost, ${ }_{451}$ without having to explore the whole solution tree. The algo452 rithm consists of two steps: a branching step and a bounding ${ }_{453}$ step. The branching step consists of exploring possible choices 454 for $s_{i+1}$ once we have made a feature selection for $s_{i}$. In other 455 words, we split the node $\left(s_{i}, f^{i}\right)$ into multiple nodes $\left(s_{i+1}, f_{k}^{i+1}\right)$, ${ }_{456}$ where $f_{k}^{i+1}$ are features in the priority list of $s_{i+1}$. The bounding ${ }_{457}$ step allows the algorithm to stop exploring a partial solution if 458 the total cost of features in the solution is higher than the cost 459 of the best solution found so far. Figure 5 illustrates the search 460 for an optimal solution, given a sketch with 3 strokes.

${ }_{461}$ It is possible for a feature to be the first choice in the prior462 ity lists for two or more strokes. To handle this, when explor${ }_{463}$ ing a possible solution, a feature curve assigned to a stroke is 464 no longer considered for subsequent strokes. Our branch and 465 bound algorithm will explore other solutions with the feature 466 curve assigned to different strokes as long these solutions are ${ }_{467}$ guaranteed to have a smaller cost than the current best solution.

\section{5.2.3. Stroke in world space}

469 The previous minimization gives us, for each stroke $s$, an 470 associated terrain feature $f$. However, the stroke $s$ has its points ${ }_{471}$ in screen space, whereas the points of $f$ are in the world space.
${ }_{472}$ Our goal is to place the stroke in the world space, in order to de473 duce terrain constraints, i.e. find the distance of their projection 474 from the camera.

475 For each point of the stroke $q_{s}=\left(x_{s}, y_{s}\right)$, we check if there ${ }_{476}$ exists a feature point $q_{f}$ whose projection on screen $q_{p}=P\left(q_{f}\right)=$ $477\left(x_{p}, y_{p}\right)$ has the same $\mathrm{x}$-coordinate as $q_{s}$, i.e. $x_{s}=x_{p}$. If this 478 point exists, we project the stroke point on the world space, us479 ing the distance of $q_{f}$ from the camera as a depth value.

480 The possible undetermined points depth, at the stroke bor${ }_{481}$ ders, are set in world space to follow the stroke tangent, in the 482 world space.

\section{${ }_{483}$ 5.3. Completing selected $3 D$ features}

$484 \quad$ Using user-specified endpoints of an occluded stroke during 485 the generation of deformation constraints would create silhou${ }_{486}$ ettes that appear to start exactly at these endpoints. This can 487 look quite unnatural when viewed from a different position than 488 the first person camera position used for sketching: indeed, the 489 endpoint of the occluded stroke (a junction) is typically above 490 the terrain and thus, a sharp deformation will be created at that 491 point.

${ }_{492}$ We address this problem by extending 3D features assigned 493 to strokes at both endpoints along their tangents, until they reach 494 the surface of the terrain. This is provided as an optional step 495 in the editing process. An example of feature completion is 496 presented in Figure 6. This simple approach only produces re497 alistic terrain silhouettes for strokes with a low-frequency struc498 ture. More sophisticated contour completion methods such as 499 the one presented in SmoothSketch [18] could alternatively be 500 used to support elaborate strokes.

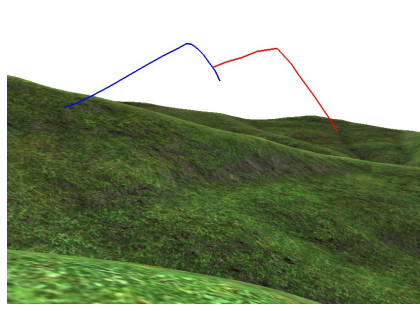

(a) User input

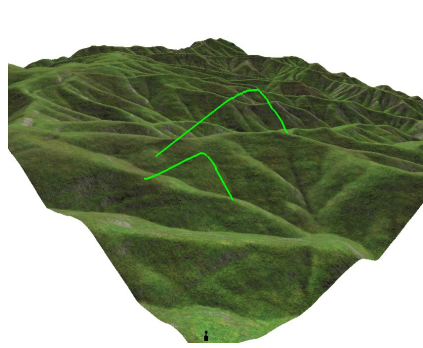

(c) Extend the matched features

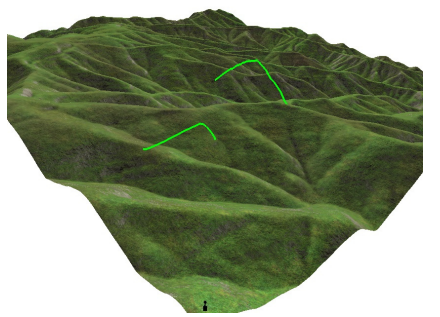

(b) Matched features

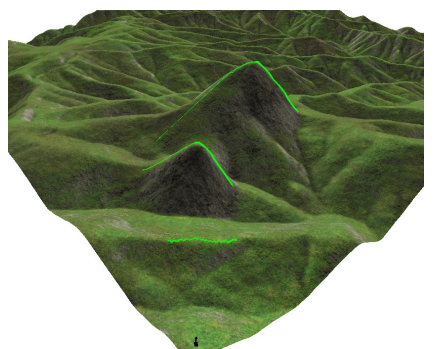

(d) Resulting terrain
Figure 6: Completing selected features: after matching 2D strokes to terrain features, we extend these features until they reach the surface of the terrain, to ensure a smooth transition from specified silhouettes to the terrain. 


\section{${ }_{501}$ 6. Terrain deformation}

502 In the previous section, we analysed terrain features and 503 used them to position the strokes in the world space. We present 504 in this section how we use them as constraints to deform the ex505 isting terrain.

\section{6.1. Diffusion-based equation solver}

507 Our deformation algorithm relies on iterative diffusion of 508 displacement constraints, which are computed from the 3D stroke 509 positioned in the world space.

510 The diffusion method, first introduced in work by Emilien 511 et al. [31], consists in computing the difference of the curve 512 height and the terrain height $\mathcal{H}$, and to diffuse these differences ${ }_{513}$ (instead of absolute height values) using a multi-grid Poisson 514 solver similar that used by Hnaidi et al. [14].

515 More precisely, for each point $p=(x, y, z)$ of the stroke in 516 the world space, we compute $\delta=z-\mathcal{H}(x, y)$, and set it as a dis517 placement constraint. The constraints are rasterised on a grid, 518 whose resolution is equal to the terrain resolution. After hav519 ing set the constraints of all strokes, we perform the diffusion, 520 which gives the displacement map $\mathcal{M}$.

${ }_{521}$ The displacement is finally applied on the terrain height ${ }_{522}$ field $\mathcal{H}$, whose feature line silhouettes are now matching the ${ }_{523}$ user strokes, when seen from the first-person viewpoint used 524 for sketching. The deformation only consists of adding the two 525 heights, $\mathcal{H}^{\prime}(x, y)=\mathcal{H}(x, y)+\mathcal{M}(x, y)$, where $\mathcal{H}^{\prime}$ is the result526 ing terrain. Because height differences are propagated, instead ${ }_{527}$ of absolute heights, the terrain preserves fine-scale details dur528 ing the deformation.

\section{6.2. Lowering protruding silhouettes}

${ }_{530}$ After deformation, the user-defined silhouettes may be hid${ }_{531}$ den by other parts of the terrain. To address this issue, we de532 tect the unwanted protruding silhouettes and constrain them to ${ }_{533}$ a lower position so that the user-defined silhouettes become vis534 ible.

\section{6.2.1. Detecting most protruding silhouette edges}

${ }_{536}$ First, all visible silhouettes are detected, with the algorithm ${ }_{537}$ discussed in Section 5.1. These silhouettes are projected onto ${ }_{538}$ the sketching plane. Let $s$ be a silhouette of the deformed land${ }_{539}$ scape, inherited from the example terrain. The mountain of sil${ }_{540}$ houette $s$ hides a user-specified silhouette $g$ if $s$ is closer to the ${ }_{541}$ camera than $g$ and the projection $s_{p}$ of $s$ in the sketching plane 542 has a higher altitude than $g_{p}$, the projection of $g$. In this case, ${ }_{543} S$ is an unwanted protruding silhouette. Determining how much $544 S$ should be lowered is done as follows: Let $h$ be the maximum 545 height difference between $s$ and a silhouette $g$ hidden by $s$. It 546 therefore follows that $h$ is the minimum altitude by which $s$ ${ }_{547}$ should be lowered to ensure the silhouettes it hides become vis548 ible. Our solution is simply to uniformly lower $s$ by an offset $h$. ${ }_{549}$ This method is applied to all unwanted protruding silhouettes 550 and we use the set of lowered silhouettes to form new deforma551 tion constraints.

\section{6.2.2. Updating deformation constraints}

${ }_{553}$ The new deformation constraints from the lowered protrud554 ing silhouettes are added to the set of constraints associated to 555 the sketched silhouettes, and the terrain is deformed once again ${ }_{556}$ using the method of Section 6.1. This operation maintains the 557 user-specified silhouettes while lowering areas around the un558 wanted protruding silhouettes, so that user specifications are 559 satisfied.

The process of detecting protruding silhouettes and using $\oint_{61}$ this information to further constrain the terrain is repeated un562 til protruding silhouettes are no longer detected. In practice, a 563 single iteration is usually sufficient to make all user-specified 564 silhouette strokes visible.

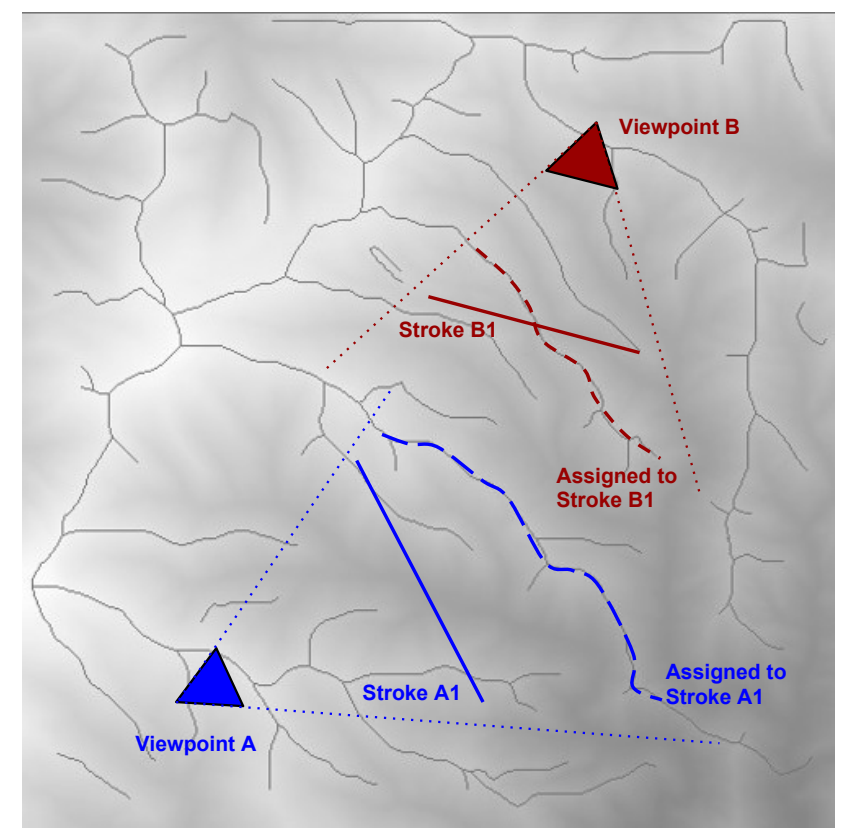

Figure 7: Multi-view from two overlapping viewpoints. Let sketch A consists of stroke A1 and sketch B consists of stroke B1. A and B are intersecting sketches since stroke $B 1$ is visible from $A$ and stroke $A 1$ is visible from $B$. If the indicated terrain features (shown in dashed lines) are assigned to each stroke and deformed to fit the user-specified heights, then either the silhouette created by $\mathrm{B} 1$ will be protruding viewed from A, or the silhouette created by A1 will be protruding viewed from B.This situation can only be avoided if the section of stroke A1 visible from B has the same height values as B1.

\section{${ }_{565}$ 7. Handling multi-view sketches}

With respect to our earlier work [21], we improve our frame567 work to support multi-view sketches from different viewpoints. ${ }_{568}$ We assume that the sketches provided by the artist do not cross 569 each other. Two sketches cross or intersect if parts of both 570 sketches are visible from the two sketching viewpoints. It would 571 be difficult to generate terrain silhouettes that match one sketch 572 and yet, are not detected as protruding from the other sketch573 ing viewpoint. Figure 7 shows an example of two intersecting 574 sketches. The problem of having silhouettes generated from 575 one sketch viewed as protruding silhouettes from a different 576 viewpoint cannot usually be solved, unless the intersecting sec577 tions have the same height values or the assigned features for 


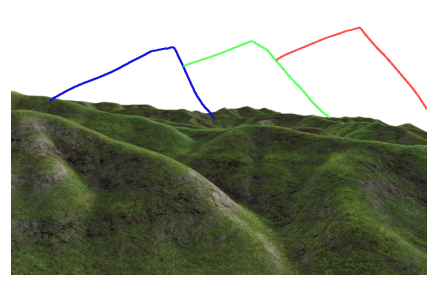

(a) Viewpoint 1

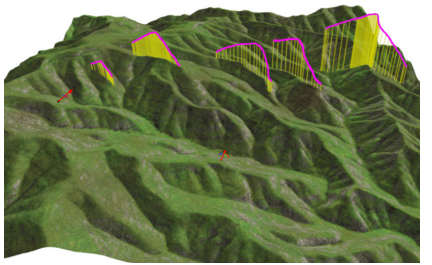

(c) Matched features (3/4 view)

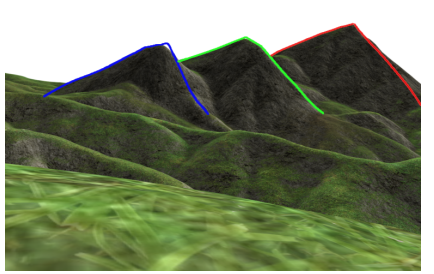

(e) Final output (viewpoint 1)

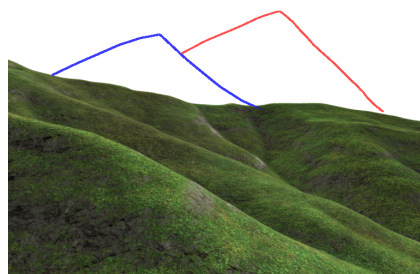

(b) Viewpoint 2

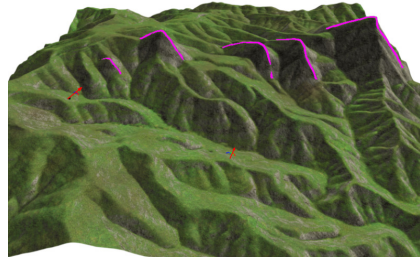

(d) Deformation based on matches

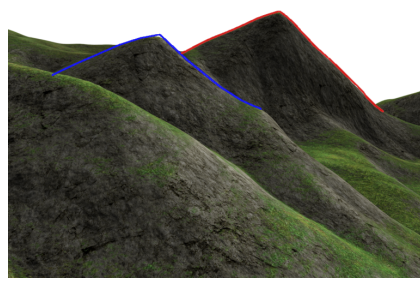

(f) Final output (viewpoint 2)
Figure 8: Sketch-based terrain editing from two different viewpoints (shown in (a) and (b)). (c) shows the terrain features assigned to each stroke, based on a modified stroke-feature matching algorithm that handles all sketches at once, while ensuring that curve constraints for different sketches do not occlude each other. The yellow lines indicate the height displacements of assigned terrain features. (d) The height displacements are used as constraints to a terrain deformation. (e,f) shows the deformed terrain from the two viewpoints. (g,h) shows the resulting terrain after lowering protruding silhouettes visible from each viewpoint.

578 one sketch are so far from the other sketch viewpoint that they 579 are not visible. Thus for overlapping viewpoints that are far 580 enough from each other, terrain features closer to the camera ${ }_{581}$ can be assigned such that no conflict occurs. Instead of includ582 ing this additional complexity to our method, we decided not to ${ }_{583}$ support intersecting sketches. We argue that in the case where 584 viewpoints are far from each other, iterative drawing can be 585 used, since the algorithm always try to assign features that are ${ }_{586}$ closer to the corresponding sketch viewpoint. Iterative drawing ${ }_{587}$ could be used for non-intersectingl multi-view sketches as well, 588 but especially in cases where multiple viewpoints are close to 589 each other, taking into account all the sketches when deciding 590 the assignment of features to strokes is important.The approach 591 discussed here provides a guarantee that each generated silhou592 ette will fit the corresponding user strokes, with no other silhou593 ette protruding when seen from the corresponding viewpoint.

${ }_{594}$ To handle non-intersecting multi-view sketches, we first pro595 cess each sketch separately by computing its stroke ordering 596 and a list of potential terrain features for each stroke. Note that
597 for each sketch, we generate this list from terrain ridges and sil598 houettes edges detected from the sketch camera position. Once 599 we have a priority list of candidate features for each stroke in 600 each sketch, we run an energy minimization process that takes 601 into consideration all the sketches at once.

602

${ }_{603}$ The energy minimization problem (Section 5.2.2) changes 604 as follows: for each input sketch $I_{t}$, we want to assign a terrain 605 feature to each of its strokes $s_{i}^{t}$ such that the total cost of all the 606 assignments is minimized, with the additional constraint that no 607 terrain feature assigned to a given stroke $s_{i}^{t}$ should fall between 608 the camera and an already assigned feature in a different sketch ${ }_{609} I_{t^{\prime}}$. This additional constraint ensures that all assigned terrain 610 features remain visible from their respective sketch viewpoint. ${ }_{611}$ To handle all sketches at once in the branch-and-bound algo612 rithm, we first explore solutions for the first drawn sketch and ${ }_{613}$ then proceed to the next one. Similarly to the process in Sec${ }_{614}$ tion 5.2.2, the list of candidates for every stroke is updated ac615 cording to constraints within each sketch and across sketches. ${ }_{616}$ This modified branch and bound scheme effectively generates 617 stroke-feature matches for all sketches.

618 Once we have assigned a terrain feature to each stroke, all ${ }_{619}$ the combined matched features are used to deform the terrain 620 (Section 6). To handle residual artifacts from the deformation, 621 we lower protruding silhouettes one sketch at a time, for all 622 sketches. Because the influence of terrain deformation is lo${ }_{623}$ calized, lowering protruding silhouettes for one sketch have a 624 limited effect on terrain silhouettes for another sketch. Figure 8 625 shows a terrain editing from two sketches, each drawn from a 626 different camera position and orientation. Note how for both ${ }_{627}$ sketches, user strokes correspond to terrain silhouettes, while 628 the whole terrain remains plausible from different viewpoints. ${ }_{629}$ This would not have been the case if the two sketches had been ${ }_{630}$ processed sequentially, since deformations due to the second 631 sketch would have likely modify silhouettes generated for the 632 first sketch.

\section{${ }_{633}$ 8. Results}

634 Validation examples. The examples below and the associated 635 video illustrate the results of our method in a variety of cases. ${ }_{636}$ In particular, Figure 9 shows editing of a terrain with a complex ${ }_{637}$ sketch containing 4 T-junctions. Our method is also able to han${ }_{638}$ dle complex mountains where ridges are not as well-defined as ${ }_{639}$ they are on smooth landscapes. An example of this is shown 640 in Figure 10. Our proposed approach differs from other sketch${ }_{641}$ based methods in that non-planar silhouettes can be generated 642 from planar user-sketched strokes. This is illustrated in Figure 643 11. Moreover, the method is robust enough to support terrains 644 with few or no features, as shown in the example given in Fig645 ure 12. Indeed if the terrain contains no features, we compute a ${ }_{646} 3 \mathrm{D}$ embedding of stroke closest to the camera by projecting the ${ }_{647}$ stroke on the drawing plane determined by the camera direc648 tion and a 3D point where the stroke touches the terrain. The ${ }_{649}$ rest of the user strokes can then be placed in 3D with respect 650 to the embedding of the first stroke, using the same technique 651 we apply to strokes with no matching features in Section 5.2.2. 


\begin{tabular}{|c||c|c|c|c|}
\hline Fig. & Features & Matching & Deformation & Silhouettes \\
\hline 1 & 0.14 & 0.24 & 0.09 & 4.9 \\
2 & 0.14 & 1.5 & 0.11 & 2.6 \\
9 & 0.15 & 0.21 & 0.10 & 2.1 \\
10 & 0.12 & 0.13 & 0.10 & 9.4 \\
11 & 0.12 & 0.04 & 0.09 & 3.4 \\
\hline
\end{tabular}

Table 1: Computation times (in seconds) for examples illustrated in this paper We show computation times of the following steps: feature extraction, strokefeature matching, terrain deformation, lowering protuding silhouettes.

${ }_{652}$ Our complex sketch-based editing framework can be imple${ }_{653}$ mented at interactive rates, as illustrated in the attached video, 654 which makes it an attractive alternative to other terrain genera655 tion/editing techniques discussed in Section 2.

${ }_{656}$ Performance. The terrain editing system is implemented in $\mathrm{C}++$ 657 and the computations are measured on an Intel ${ }^{\circledR}$ Xeon ${ }^{\circledR}$ E5${ }_{658} 1650 \mathrm{CPU}$, running at $3.20 \mathrm{GHz}$ with $16 \mathrm{~GB}$ of memory. We ${ }_{659}$ present the computation times of resuts illustrated in this paper ${ }_{660}$ in the Table 1. The feature extraction and terrain deformation ${ }_{661}$ computation times only depend on the terrain resolution, which 662 is $512 \times 512$ in the examples. Feature matching performance ${ }_{663}$ depends on the number of strokes and the number of extracted ${ }_{664}$ features. In our examples, the average number of extracted fea665 tures was around 1000 and mostly consisted of short terrain sil${ }_{666}$ houette features. The most expensive algorithm is the lowering ${ }_{667}$ protruding silhouettes, due to the expensive sihouette detection. ${ }_{668}$ Our naive implementation of silhouette detection could be op669 timised to significantly impact the overall performance of our 670 algorithm. The stroke ordering algorithm has a negligible com671 putation time. The average manual editing time was less than a 672 minute.

${ }_{673}$ Comparing feature-based constraints against planar curve con674 straints. Typical sketch-based terrain deformation techniques $675[13,27,15]$ use planar curve constraints computed from user 676 strokes. Such planar curves can be obtained by computing the 677 drawing plane from the user sketch and projecting strokes on 678 this plane to obtained their 3D position in world coordinates. 679 The normal to the drawing plane is the camera view direction 680 and one point on this plane is obtained by computing the world ${ }_{681}$ coordinates of a stroke point touching the terrain. We argue 682 that using such planar curve constraints for terrain editing pro683 duce inferior results, compared to the use of feature-based con${ }_{684}$ straints. To illustrate this, we compared the two different defor685 mation schemes, our method and the standard method, on three ${ }_{686}$ different input. Each input consists of a real landscape and a 687 one-stroke sketch drawn from a first person perspective view 688 (see Figure 13). Our method uses the matched terrain features 689 obtained from Section 5 as deformation constraints. The stan${ }_{690}$ dard method simply uses curve constraints obtained by project691 ing user strokes on the drawing plane. Figure 13 shows the 3D 692 constraints used in the terrain deformation and the final terrain 693 produced by each method. Note that the final terrain is gen694 erated by first deforming the input terrain with feature-based 695 constraints or planar curve constraints, and then lowering pro-
696 truding silhouettes. In the case of planar curve constraints, this ${ }_{697}$ last step generates non planar silhouettes, which is already an 698 improvement since the main pitfall of the standard method is 699 that it produces unrealistic mountains with planar silhouettes. 700 Even after this improvement, note how landscapes produced by 701 the standard method have more prominent silhouettes in front 702 of the user-specified silhouettes and thus may not reflect the 703 user intent. This happens when a planar curve constraint is be704 hind a terrain feature and thus the deformation raises the terrain 705 feature making it a prominent silhouette. In contrast our pro706 posed method is feature-aware and by generating deformation 707 constraints based on terrain features, reduces the risk of promi708 nent silhouettes appearing in front of user-specified silhouettes. 709 In addition, the silhouettes we generate are non-planar, since 710 they are matched with the depth of the associated terrain fea711 tures (Figure 13(h, i)). This makes the resulting terrains look ${ }_{712}$ much more natural when seen from above.

${ }_{713}$ User tests. We performed an informal user test on our single 714 viewpoint system with two experienced computer artists. The 715 system was briefly introduced to the users, who had no prior 716 knowledge of it. They were asked to draw sketches to deform 717 existing terrains. Both of them reported that our system was 718 very easy to learn and use, and were able to quickly create new ${ }_{719}$ sceneries. Their feedback indicated that the approach is origi720 nal, and seems a promising way to create a scene that matches 721 their artistic intend. These first users also asked for the abil722 ity to move within the scene and edit the terrain from multiple 723 viewpoints. This led to the work described in Section 7. Lastly, 724 the users emphasised the importance of the realistic resulting 725 terrain, and noted that it matched their sketches in the expected 726 way.

727 Limitations. Although our system succeeds in matching a com728 plex user-sketch through a natural deformation of the terrain, 729 based on its existing features, the lack of predictability of the 730 stroke-feature solver may be a problem. It is often not clear ${ }_{731}$ during the drawing stage which terrain feature will be assigned 732 to a stroke. The artist may draw a stroke with the intention of 733 turning a large-scale feature into a terrain silhouette, but the al${ }_{734}$ gorithm chooses to deform a different terrain feature instead. To 735 address this, we could also improve our matching method us736 ing extra error functions, that take into account the placement 737 of user strokes relative to the projection of terrain features on 738 the drawing plane.

739 The editing framework is also limited in the type of strokes 740 drawned and the type of terrain. For instance landscapes with ${ }_{741}$ high frequency details and a complex style such as the Grand ${ }_{742}$ Canyon are particularly difficult to edit since depending on the 743 nature of the strokes and which features are assigned to strokes, 744 the deformed region can differ significantly from the other. In 745 general, elaborate strokes that are unlikely to be terrain silhou${ }_{746}$ ette, except from a specific viewpoint, often cause several iter747 ations of terrain deformation in the neighbourhood of the as748 signed features, that either do not suceed in removing all pro749 truding silhouettes or look unrealistic when viewed from a dif750 ferent viewpoint. 
751 Another limitation comes from our deformation solver. The 752 diffusion-based deformation method sometimes creates small 753 declivities around the extremity of a constraint curve, when the 754 slope of the curve is high and the extremity is located on the 755 terrain: in this case, the terrain locally inflates, except at this 756 end-point where the deformation is zero, which causes the prob757 lem. Using an inverse distance to deform a terrain [32] does not 758 work either, because of our use of curves as constraints. Future 759 work still needs to be done on terrain deformation, especially 760 for curve-based deformations.

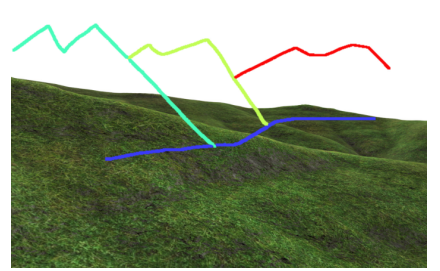

(a) User input

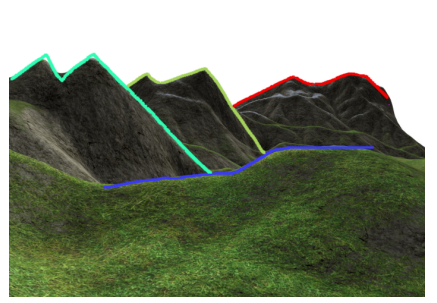

(c) Deformed terrain

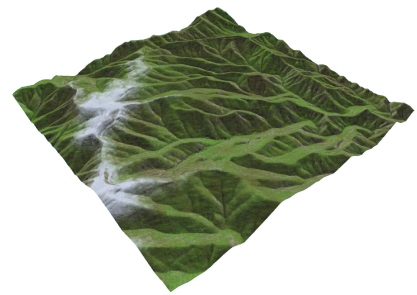

(b) Existing terrain

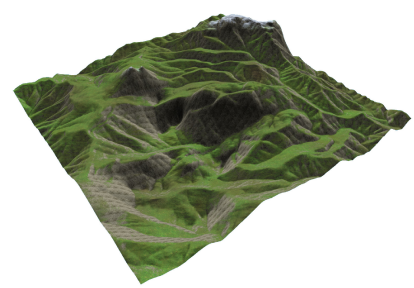

(d) Viewed from a different point

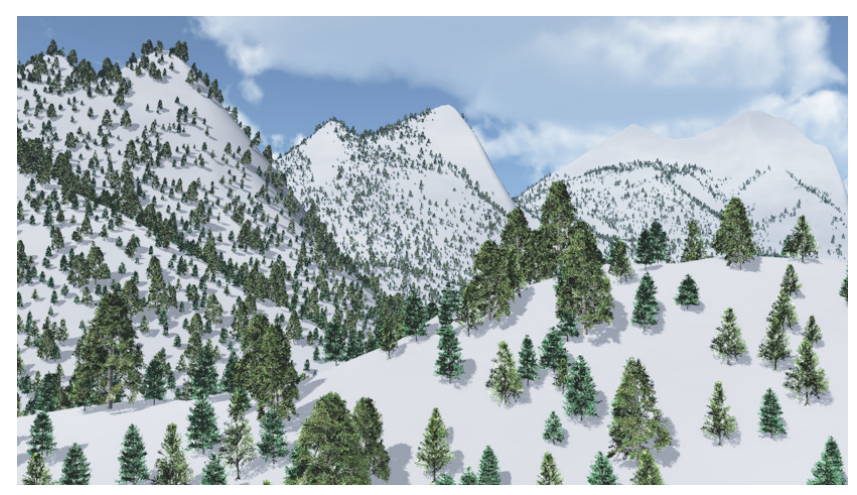

(e)

Figure 9: Terrain editing from a complex user sketch.

\section{9. Conclusion}

762 We presented a sketch-based modelling method enabling 763 the deformation of a terrain from a single viewpoint, and then ${ }_{764}$ extended it to handle multiple viewpoints simultaneously. The 765 user sketches a few silhouette strokes forming a graph with ${ }_{766} \mathrm{~T}$-junctions, similar to the silhouette representations used in 767 artistic terrain sketching. A key feature of our method is that 768 sketched silhouettes are matched with existing terrain features: 769 this enables our technique to both match silhouette strokes with

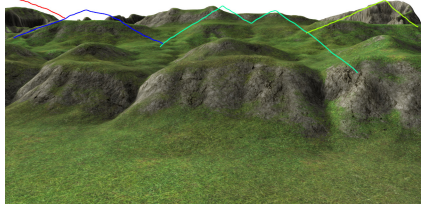

(a) User input

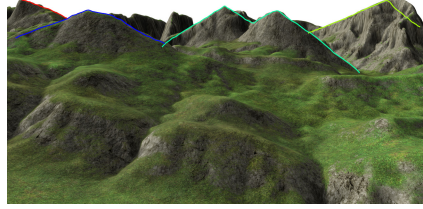

(b) Result

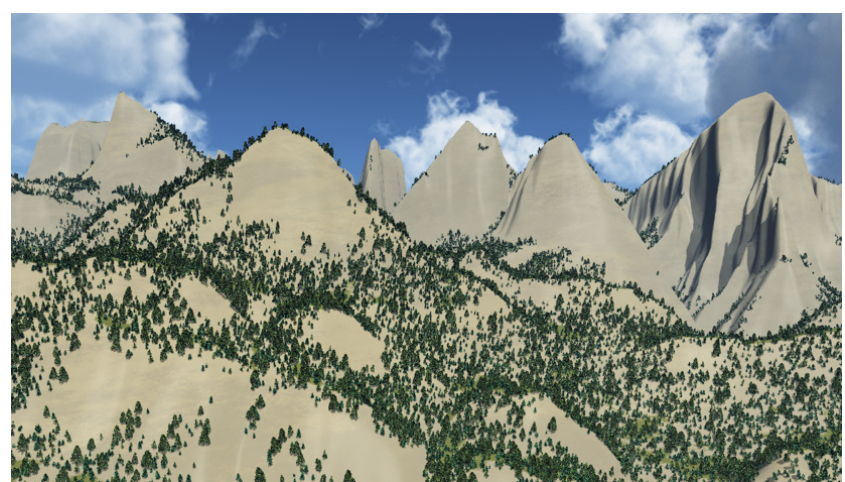

(c)
Figure 10: Editing a complex rocky mountain from a complex sketch.

770 a non-planar curve, and produce a deformation that does not 771 spoil plausibility, since the structure of ridges and valleys typi772 cally remains unchanged.

\section{Acknowledgements}

774 This work was conducted during an internship of Flora Pon775 jou Tasse at Inria Rhône-Alpes in Grenoble. It was partly sup776 ported by the ERC advanced grant EXPRESSIVE.

\section{References}

[1] Fournier A, Fussell DS, Carpenter LC. Computer rendering of stochastic models. Communications ACM 1982;25(6):371-84.

[2] Miller GSP. The definition and rendering of terrain maps. SIGGRAPH Comput Graph 1986;20(4):39-48.

[3] Lewis JP. Generalized stochastic subdivision. ACM Trans Graph 1987;6(3):167-90.

[4] Musgrave FK, Kolb CE, Mace RS. The synthesis and rendering of eroded fractal terrains. In: Proceedings of the 16th Annual Conference on Computer Graphics and Interactive Techniques. SIGGRAPH '89; New York, NY, USA: ACM; 1989, p. 41-50.

[5] Roudier P, Peroche B, Perrin M. Landscapes synthesis achieved through erosion and deposition process simulation. Computer Graphics Forum 1993;12(3):375-83.

[6] Chiba N, Muraoka K, Fujita K. An erosion model based on velocity fields for the visual simulation of mountain scenery. Journal of Visualization and Computer Animation 1998;9(4):185-94.

[7] Nagashima K. Computer generation of eroded valley and mountain terrains. Visual Computer 1997;13(9-10):456-64.

[8] Neidhold B, Wacker M, Deussen O. Interactive physically based fluid and erosion simulation. Natural Phenomena 2005;:25-32.

[9] Kristof P, Benes B, Krivanek J, Stava O. Hydraulic erosion using smoothed particle hydrodynamics. Computer Graphics Forum 2009;28(2):219-28.

[10] Cohen JM, Hughes JF, Zeleznik RC. Harold: A world made of drawings. In: Proceedings of the 1st International Symposium on Nonphotorealistic Animation and Rendering. NPAR '00; New York, NY, USA: ACM; 2000, p. 83-90. 


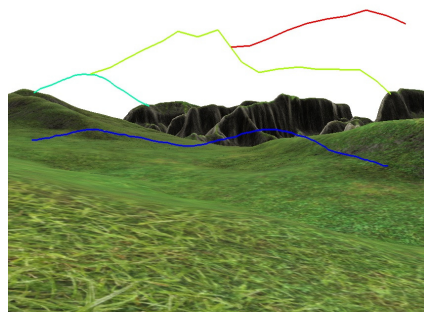

(a) User input

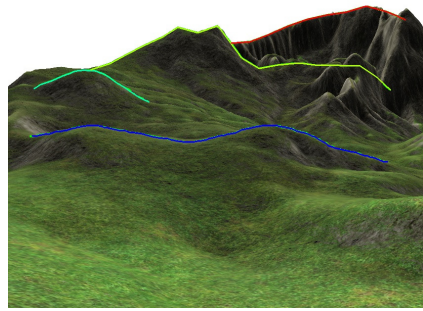

(c) Deformed terrain

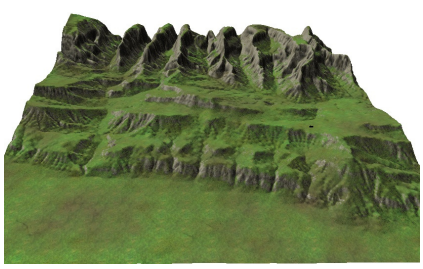

(b) Existing terrain

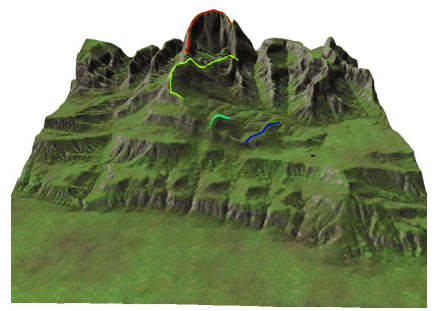

(d) Result viewed from a different point
Figure 11: Terrain editing produces non-planar silhouettes in the output, from 2D planar strokes.

[11] Watanabe N, Igarashi T. A sketching interface for terrain modeling. In: ACM SIGGRAPH 2004 Posters. SIGGRAPH '04; New York, NY, USA: ACM; 2004, p. 73-.

[12] Zhou H, Sun J, Turk Gbb, Rehg Jbb. Terrain synthesis from digital elevation models. IEEE Transactions on Visualization and Computer Graphics 2007;13(4):834-48.

[13] Gain J, Marais P, Straer W. Terrain sketching. In: Proceedings of I3D 2009: The 2009 ACM SIGGRAPH Symposium on Interactive 3D Graphics and Games. 2009, p. 31-8.

[14] Hnaidi H, Guerin E, Akkouche S, Peytavie A, Galin E. Feature based terrain generation using diffusion equation. Computer Graphics Forum 2010;29(7):2179-86.

[15] Tasse F, Gain J, Marais P. Enhanced texture-based terrain synthesis on graphics hardware. Computer Graphics Forum 2012;31(6):1959-72.

[16] Genevaux JD, Galin E, Guerin E, Peytavie A, Benes B. Terrain generation using procedural models based on hydrology. ACM Transactions on Graphics 2013;32(4).

[17] Dos Passos V, Igarashi T. Landsketch: A first person point-of-view example-based terrain modeling approach. In: Proceedings - SketchBased Interfaces and Modeling, SBIM 2013 - Part of Expressive 2013. 2013, p. 61-8.

[18] Karpenko O, Hughes J. Smoothsketch: 3d free-form shapes from complex sketches. In: ACM SIGGRAPH 2006 Papers, SIGGRAPH '06. 2006, p. 589-98.

[19] Singh K, Fiume E. Wires: A geometric deformation technique. In: Proceedings of the 25th Annual Conference on Computer Graphics and Interactive Techniques. SIGGRAPH '98; New York, NY, USA: ACM; 1998, p. 405-14.

[20] Zimmermann J, Nealen A, Alexa M. Silsketch: Automated sketch-based editing of surface meshes. In: Proceedings of the 4th Eurographics Workshop on Sketch-based Interfaces and Modeling. SBIM '07; New York, NY, USA: ACM; 2007, p. 23-30.

[21] Tasse FP, Emilien A, Cani MP, Hahmann S, Bernhardt A. First person sketch-based terrain editing. In: Proceedings of the 2014 Graphics Interface Conference. GI '14; Canadian Information Processing Society; 2014, p. 217-24.

[22] Natali M, Lidal EM, Viola I, Patel D. Modeling terrains and subsurface geology. In: Proceedings of EuroGraphics 2013 State of the Art Reports (STARs). Eurographics; Eurographics 2013 - State of the Art Reports; 2013, p. 155-73.

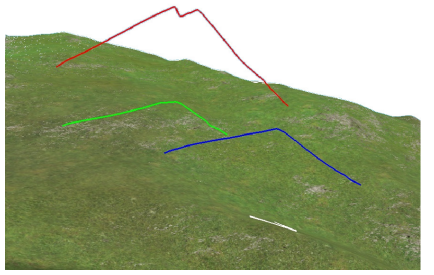

(a) User input

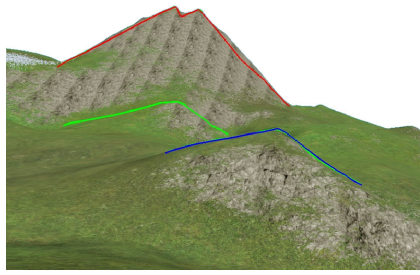

(b) Result

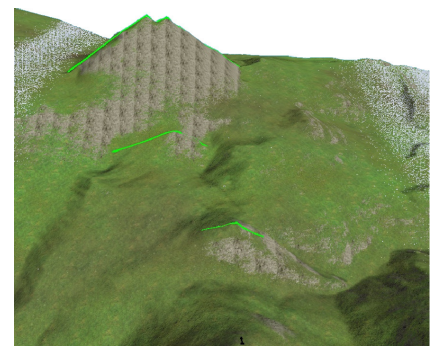

(c) View from another point

Figure 12: Adding deformation constraints automatically: the stroke furthest away from the user did not have an assigned feature to it and so one was automatically generated, and positioned on a plane orthogonal to the view direction, such that stroke ordering is respected.

[23] Mandelbrot BB. The fractal geometry of nature. New York: W. H. Freeman; 1983.

[24] Ebert DS, Musgrave FK, Peachey D, Perlin K, Worley S. Texturing and Modeling: A Procedural Approach. 3rd ed.; San Francisco, CA, USA: Morgan Kaufmann Publishers Inc.; 2002.

[25] Kelley AD, Malin MC, Nielson GM. Terrain simulation using a model of stream erosion. Computer Graphics (ACM) 1988;22(4):263-8.

[26] McCrae J, Singh K. Sketch-based path design. In: Proceedings of Graphics Interface 2009. GI '09; Toronto, Ont., Canada, Canada: Canadian Information Processing Society; 2009, p. 95-102.

[27] Bernhardt A, Maximo A, Velho L, Hnaidi H, Cani MP (IMPA, Rio de Janeiro, Brésil). Real-time Terrain Modeling using CPU-GPU Coupled Computation. In: XXIV SIBGRAPI. Maceio, Brazil; 2011,

[28] Chang YC, Sinha G. A visual basic program for ridge axis picking on dem data using the profile-recognition and polygon-breaking algorithm. Computers and Geosciences 2007;33(2):229-37.

[29] Bangay S, de Bruyn D, Glass K. Minimum spanning trees for valley and ridge characterization in digital elevation maps. In: Proceedings of the 7th International Conference on Computer Graphics, Virtual Reality, Visualisation and Interaction in Africa. AFRIGRAPH '10; New York, NY, USA: ACM; 2010, p. 73-82.

[30] Clausen J. Branch and bound algorithms-principles and examples. Parallel Computing in Optimization 1997;:239-67.

[31] Emilien A, Poulin P, Cani MP, Vimont U. Interactive procedural modeling of coherent waterfall scenes. Computer Graphics Forum 2014; To appear.

[32] Jenny H, Jenny B, Cartwright WE, Hurni L. Interactive local terrain deformation inspired by hand-painted panoramas. Cartographic Journal, The 2011;48(1):11-20. 


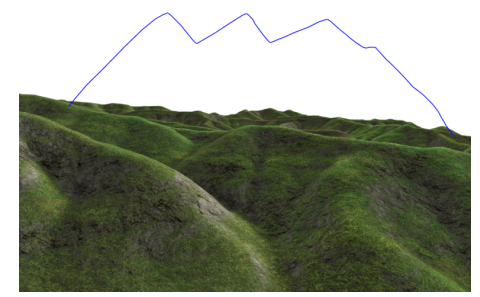

(a) Example 1

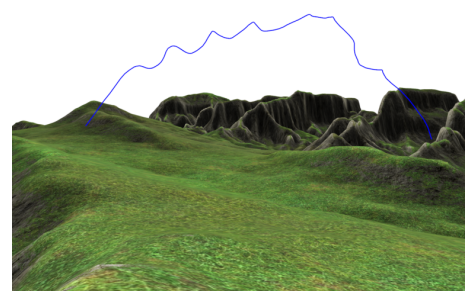

(b) Example 2

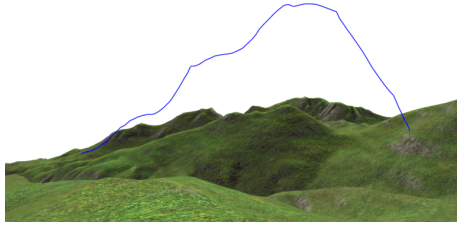

(c) Example 3

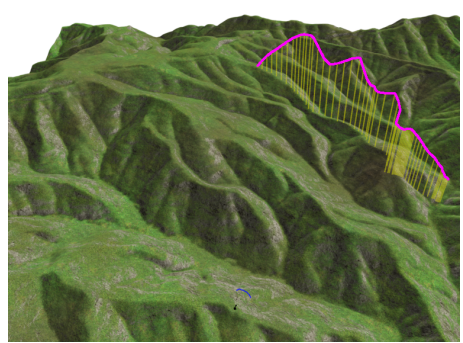

(d) Example 1, feature-based constraints

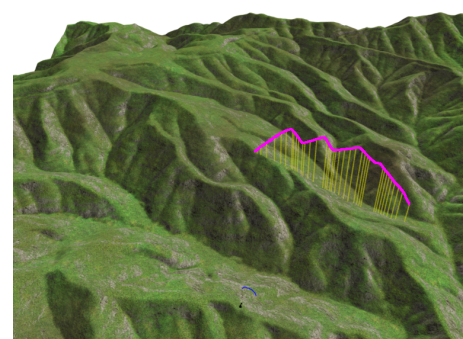

(e) Example 1, planar curve constraints

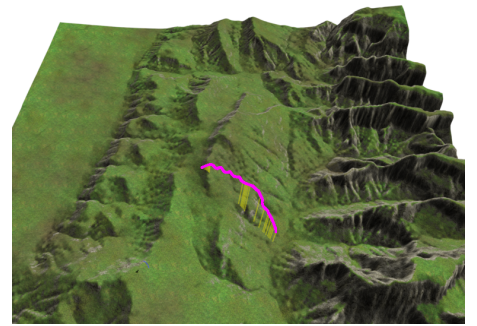

(i) Example 2, planar curve constraints

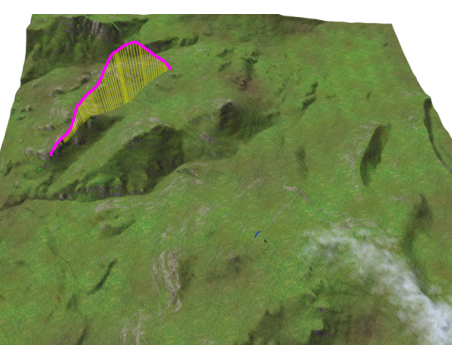

(m) Example 3, planar curve constraints

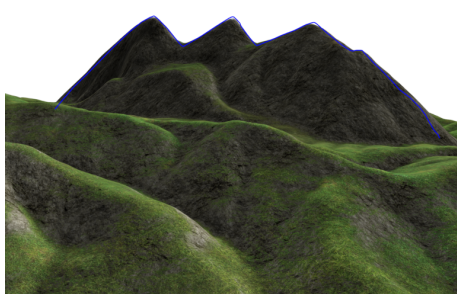

(f) Example 1, our method: result

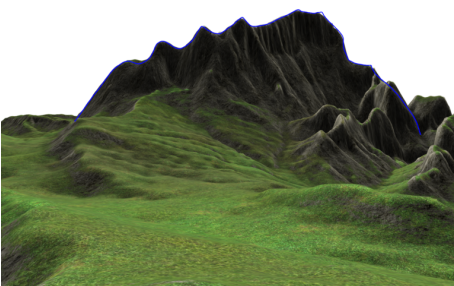

(j) Example 2, our method: result

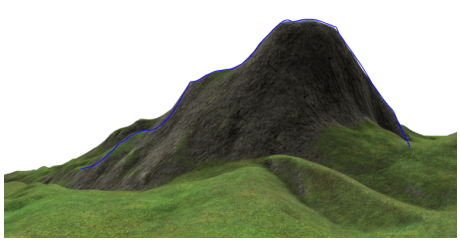

(n) Example 3, our method: result

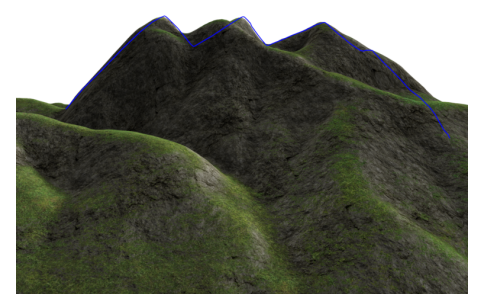

(g) Example 1, standard method: result

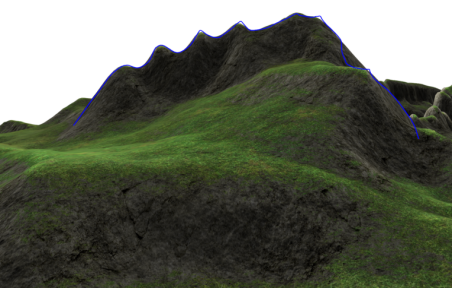

(k) Example 2, standard method: result

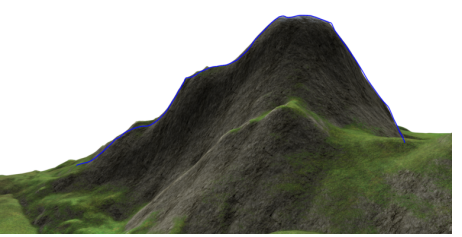

(o) Example 3, standard method: result

(1) Example 3, feature-based constraints

Figure 13: Comparing terrain deformation with feature-based constraints (our method) against editing from planar curve constraints (standard method). The final output produced by our deformation scheme has less prominent terrain silhouettes appearing between the camera position and the user-specified silhouettes, and thus is closer to the user intent. 